\title{
Lesão renal aguda na COVID-19 e abordagem multiprofissional
}

\author{
Acute kidney injury at COVID-19 and a multiprofessional approach \\ Lesión renal aguda en COVID-19 y un enfoque multiprofesional
}

Recebido: 01/03/2021 | Revisado: 06/03/2021 | Aceito: 18/03/2021 | Publicado: 26/03/2021

Ana Caroline Gusmão de Matos ORCID: https://orcid.org/0000-0002-4451-0243

Universidade Tiradentes, Brasil

E-mail: anagusmaao@gmail.com

Thiago Vaz de Andrade

ORCID: https://orcid.org/0000-0001-7950-4073

Universidade Tiradentes, Brasil

E-mail: thiagovazzandrade@gmail.com

Nathalia Costa Macedo Noronha

ORCID: https://orcid.org/0000-0003-0801-1217

Universidade Tiradentes, Brasil

E-mail: macedoacad@gmail.com

Thayná Bezerra Lima

ORCID: https://orcid.org/0000-0001-7574-8820

Universidade Tiradentes, Brasil

E-mail: thaynalima34@hotmail.com

Carmem Isaura Salles do Nascimento

ORCID: https://orcid.org/0000-0002-3000-8340

Universidade Tiradentes, Brasil

E-mail: carmem.salles9@gmail.com

Alícia Calinne Melo Santos

ORCID: https://orcid.org/0000-0001-9173-4760

Universidade Tiradentes, Brasil

E-mail: aliciacalinne@gmail.com

Valéria Soares de Jesus Santana

ORCID: https://orcid.org/0000-0001-6558-0534 Universidade Tiradentes, Brasil

E-mail: valeriiasantana@ hotmail.com

Luana Godinho Maynard

ORCID: https://orcid.org/0000-0003-0950-4742 Universidade Tiradentes, Brasil E-mail: lgmayfisio@gmail.com

Carla Viviane Freitas de Jesus ORCID: https://orcid.org/0000-0002-7775-6610 Universidade Tiradentes, Brasil E-mail: carlavfj@gmail.com

\begin{abstract}
Resumo
Objetivo: Identificar a relação da COVID-19 com a lesão renal aguda, bem como o papel da abordagem multiprofissional nesse cenário. Metodologia: Trata-se de uma revisão narrativa realizada a partir das bases de dados PubMed, SciELO e LILACS, utilizando os descritores "COVID-19" e "Acute Kidney Injury". Foram incluídos estudos originais publicados disponíveis na íntegra para leitura, publicados nos últimos 5 anos, nos idiomas português, inglês e/ou espanhol. Foram excluídos artigos veterinários, os que avaliaram pacientes com doenças renais crônicas prévias à infecção pela COVID-19 ou transplantados, além de resumos isolados e editoriais. Resultados: O SARSCoV-2 invade células humanas a partir da enzima conversora de angiotensina 2 e serino-proteases transmembrana tipo 2, ocasionando resposta inflamatória sistêmica por tempestade de citocinas, efeito citotrópico direto induzido por vírus e necrose tubular aguda, devido a falha de múltiplos órgãos e choque. A rabdomiólise contribui para esse quadro. Clinicamente, os pacientes com lesão renal aguda e COVID-19 podem ser apresentar com sintomas geniturinários, diarreia, proteinúria, hematúria e alterações de ureia e creatinina. A abordagem ao paciente nefrológico no contexto pandêmico envolve reforço de medidas preventivas ao novo vírus, gestão de saúde eficaz e colaboração entre médicos e enfermeiros de diversas especialidades, em especial intensivistas e nefrologistas. Conclusão: A infecção pelo SARS-CoV-2 pode ocasionar lesão renal aguda por toxicidade viral direta ou desregulação de mecanismos imunológicos. O quadro clínico nesses casos é marcado por sintomas de falência renal e infecção. A atuação sinérgica de equipe de saúde multiprofissional e gestores de saúde é fundamental nesse cenário.
\end{abstract}

Palavras-chave: Equipe de assistência ao paciente; Fenômenos fisiológicos; Infecções por coronavirus; Lesão renal aguda. 


\begin{abstract}
Aim: To identify the relationship between COVID-19 and acute kidney injury, as well as the role of the multiprofessional approach in this scenario. Methodology: This is a narrative review carried out from the PubMed, SciELO and LILACS databases, using the descriptors "COVID-19" and "Acute Kidney Injury". The inclusion criteria were: original published studies available in full for reading, published in the last 5 years, in Portuguese, English and / or Spanish. Veterinary articles were excluded, as well as those that evaluated patients with chronic kidney diseases previous to infection by COVID-19 or transplanted patients, isolated abstracts and editorial letters. Results: SARSCoV-2 uses angiotensin 2-converting enzyme and type 2 transmembrane serine proteases to invade human cells, causing a systemic inflammatory response because of cytokine storm; direct cytotropic effect induced by virus; and acute tubular necrosis, due to multiple organ failure and shock. Rhabdomyolysis contributes to this condition. Clinically, patients with acute kidney injury and COVID-19 may present genitourinary symptoms, diarrhea, proteinuria, hematuria and changes in urea and creatinine. The approach to the nephrological patient in a pandemic context involves reinforcement of preventive measures against the new virus, effective health management and collaboration between doctors and nurses of different specialties, especially intensivists and nephrologists. Conclusion: SARS-CoV-2 infection can cause acute kidney injury due to direct viral toxicity or dysregulation of immunological mechanisms. The clinical picture in these cases is marked by symptoms of kidney failure and infection. The synergistic performance of the multiprofessional health team and health managers is essential in this scenario.
\end{abstract}

Keywords: Acute kidney injury; Coronavirus infections; Patient care team; Physiological phenomena.

\title{
Resumen
}

Objetivo: Identificar la relación entre COVID-19 y la lesión renal aguda, así como el papel del abordaje multiprofesional en este escenario. Metodología: Se trata de una revisión narrativa realizada a partir de las bases de datos PubMed, SciELO y LILACS, utilizando los descriptores "COVID-19" y "Acute Kidney Injury". Se incluyeron estudios originales publicados disponibles en su totalidad para lectura, publicados en los últimos 5 años, en portugués, inglés y / o español. Se excluyeron los artículos veterinarios, aquellos que evaluaban pacientes con enfermedades renales crónicas previas a la infección por COVID-19 o trasplantados, además de los resúmenes aislados y editoriales. Resultados: El SARS-CoV-2 invade las células humanas de la enzima convertidora de angiotensina 2 y serina proteasas transmembrana tipo 2, provocando una respuesta inflamatoria sistémica por tormenta de citoquinas, efecto citotrópico directo inducido por virus y necrosis tubular aguda, debido a falla orgánica múltiple y conmoción. La rabdomiólisis contribuye a esta condición. Clínicamente, los pacientes con insuficiencia renal aguda y COVID-19 pueden presentar síntomas genitourinarios, diarrea, proteinuria, hematuria y cambios en la urea y la creatinina. El abordaje del paciente nefrológico en un contexto pandémico implica el refuerzo de las medidas preventivas frente al nuevo virus, una gestión sanitaria eficaz y la colaboración entre médicos y enfermeras de diferentes especialidades, especialmente intensivistas y nefrólogos. Conclusión: La infección por SARS-CoV-2 puede causar una lesión renal aguda debido a la toxicidad viral directa o la desregulación de los mecanismos inmunológicos. El cuadro clínico en estos casos está marcado por síntomas de insuficiencia renal e infección. El desempeño sinérgico del equipo de salud multiprofesional y los gerentes de salud es fundamental en este escenario.

Palabras clave: Fenómenos fisiológicos; Grupo de atención al paciente; Infecciones por coronavirus; Lesión renal aguda.

\section{Introdução}

Em dezembro de 2019, um surto de pneumonia de causa desconhecida em Wuhan, província de Hubei, China, mobilizou os cientistas chineses, que isolaram o vírus SARS-CoV-2 em janeiro do ano seguinte. A doença ocasionada pelo novo coronavírus recebeu o nome de COVID-19 e se espalhou rapidamente pela China e países em todo o mundo. Diante disso, a Organização Mundial da Saúde (OMS) declarou oficialmente a pandemia como uma emergência de saúde pública de interesse internacional em 30 de janeiro de 2020 (Cui et al., 2020; Ulu et al., 2020; Na et al., 2020). O principal modo de infecção é a transmissão inter-humana por meio da pulverização de gotículas de um indivíduo infectado através da tosse ou espirro (D-Marco et al., 2020). A infecção foi relatada em todas as idades, mas estudos epidemiológicos recentes mostraram que idosos com doenças crônicas provavelmente têm um curso grave (Cui et al., 2020; D-Marco et al., 2020; Ulu et al., 2020).

No que tange à fisiopatologia da COVID-19, uma tempestade de citocinas é desencadeada devido ao poder que o vírus tem de atenuar o efeito antiviral do interferon (IFN) tipo I, resultando em replicação viral descontrolada, com o consequente influxo de neutrófilos e monócitos/macrófagos e hiperprodução de citocinas pró-inflamatórias (D-Marco et al., 2020). 
Os mecanismos de infecção do SARS-CoV-2 têm sido apontados como risco potencial para lesão renal aguda (LRA). A literatura mostra que células epiteliais tubulares proximais renais cultivadas, células mesangiais glomerulares e podócitos que expressam enzima conversora de angiotensina 2 (ECA2) em sua superfície, podem representar um alvo para COVID-19 (Zou et al., 2020).

A possibilidade de uma "nefropatia por SARS-CoV-2" é levantada através de relatos de anormalidades urinárias em pacientes com COVID-19, coloração positiva de túbulos com antígenos virais e componentes do complemento em uma série autóptica, visualização de partículas virais em células epiteliais tubulares e podócitos no exame ultraestrutural em outras biópsias renais e isolamento de SARS-CoV-2 na urina (Farouk et al., 2020).

Diante da influência do novo coronavírus sobre os mais diversos sistemas, bem como considerando suas implicações nas funções renais e a elevada morbimortalidade ocasionada por esses danos, surge a demanda por políticas de gerenciamento atualizadas para os cuidados nefrológicos, com participação da equipe multiprofissional (Ulu et al., 2020). O objetivo do presente estudo foi identificar a relação da COVID-19 com a lesão renal aguda, bem como a abordagem multiprofissional diante desses casos.

\section{Metodologia}

Trata-se de uma revisão narrativa da literatura com natureza qualitativa, conforme Pereira et al. (2018). A busca de artigos foi realizada nas bases de dados Medical Publications (PubMed), Scientific Electronic Library Online (SciELO) e Literatura Latino-Americana e do Caribe em Ciências da Saúde (LILACS), a partir dos descritores "Acute Kidney Injury" e "COVID-19", utilizando operador booleano AND.

Após a aplicação de filtros e exclusão de repetições, procedeu-se a leitura exploratória de títulos e resumos. Os artigos elegíveis tiveram seus resumos lidos e, posteriormente, texto lido na íntegra.

Como critério de inclusão, foram adotados: estudos originais publicados disponíveis na íntegra para leitura, publicados nos últimos 5 anos, nos idiomas português, inglês e/ou espanhol. Foram excluídos os artigos veterinários, os que incluíram pacientes com doenças renais crônicas prévias à infecção pela COVID-19 ou transplantados, além de resumos isolados e editoriais.

\section{Resultados e Discussão}

\subsection{Estatísticas da lesão renal aguda na COVID-19}

A incidência de LRA em pacientes infectados por COVID-19 é de 0 (WANG et al., 2020b) a 29\% (Yang et al., 2020). A ampla variação de incidência se deve às inconsistências na definição de LRA e às diferenças de amostra dos estudos, que vão de pacientes com condições leves da doença a hospitalizados em estado crítico. Estudos de Zhou et al. (2020) e Zhang et al. (2020) a taxa de incidência variou de 14,5\% a 50\% em pacientes com infecção grave pelo SARS-CoV-2 admitidos na unidade de terapia intensiva (UTI). Nos casos de lesão significativa, a terapia renal substitutiva pode ser empregada, tratamento este necessário em cerca de 3,2\% dos pacientes hospitalizados com COVID-19 (Richardson et al., 2020).

\subsection{Etiologia da lesão renal aguda em pacientes infectados pelo SARS-CoV-2}

A LRA na infecção por COVID-19 se deve à combinação de resposta inflamatória sistêmica induzida por citocinas, efeito citotrópico direto induzido por vírus e necrose tubular aguda, devido a falha de múltiplos órgãos e choque. Outros fatores como depleção de volume, toxicidade do medicamento, exposição ao contraste e hipotensão podem resultar em insuficiência renal aguda (IRA) (Chenna et al., 2020).

O passo inicial da infecção por SARS-CoV-2 é invadir células humanas. SARS-CoV-2 e SARS-CoV possuem um 
ancestral comum similar ao coronavírus do morcego HKU9-1. Esses vírus utilizam receptores ECA2 e serino-proteases transmembrana tipo 2 (TMPRSS2) para entrar na célula humana (HOFFMANN et al., 2020). A proteína Spike (S) do vírus tem alta afinidade de ligação ao domínio catalítico da ECA2 (Hoffmann et al., 2020), o que pode causar alterações conformacionais na proteína S (Wrapp et al., 2020). As proteases celulares (TMPRSS2) realizam o priming e clivagem da proteína $\mathrm{S}$ e, com isso, permitem a fusão das membranas virais e celulares (Hoffmann et al., 2020), mecanismo central na determinação do tropismo tecidual (Chenna et al., 2020). Wrapp et al. (2020) demonstraram que, na microscopia crioeletrônica, a proteína S do nCoV tem afinidades 10 a 20 vezes maiores para a ECA2 quando comparada com a do SARS-CoV.

A infecção afeta principalmente o trato respiratório inferior, mas também pode ser apresentado com envolvimento de vários órgãos. A análise de sequenciamento de RNA de célula única mostrou que os receptores ECA2 estavam localizados nas células alveolares tipo 2, células do miocárdio, células dos túbulos proximais dos rins, células epiteliais do esôfago e células uroepiteliais da bexiga. Desta forma, pulmões, coração, esôfago, rim, bexiga ou íleo tornam-se os órgãos-alvo do COVID-19 (Ulu et al., 2020; Xu et al., 2020).

A expressão do gene para ECA2 é 100 vezes maior no tecido renal em comparação com o pulmão (Li et al., 2020a). Nos túbulos renais, há expressão significativa de ECA2. Além disso, receptores ECA 2 são encontrados também nos podócitos, apesar de em menor proporção, o que pode explicar a propensão do vírus infectar essas células (Gupta et al., 2020).

Após a invasão celular pelo vírus, os mecanismos para lesão renal têm início, a saber: desregulação da angiotensina, ativação da via imune inata e adaptativa e hipercoagulação (Farouk et al., 2020).

Em condições fisiológicas o angiotensinogênio é convertido em angiotensina I pela renina, que por sua vez é convertida em angiotensina II (Ang II) pela ECA. A angiotensina II ativa o receptor da angiotensina tipo 1 (AT1R) e a aldosterona e exerce vasoconstrição e retenção de sódio, o que resultaria em hipertensão. Entretanto a ECA2 converte a angiotensina II em angiotensina (Ang)-(1-7), que exerce efeitos vasodilatadores e regula negativamente o sistema RAS (Silva et al., 2013). Na ausência e desregulação da ECA2, a angiotensina II causa inflamação, fibrose e edema por meio do AT1R o que resulta na síndrome do desconforto respiratório agudo grave.

Mecanismos imunológicos também atuam nesse processo. Em indivíduos saudáveis, a resposta imune inata contra a infecção viral depende muito das respostas do interferon (IFN) tipo I e sua cascata que culmina no controle da replicação viral e na indução de uma resposta imune adaptativa eficaz. Os coronavírus podem atenuar o efeito antiviral do IFN tipo I, resultando em replicação viral descontrolada, com o consequente influxo de neutrófilos e monócitos/macrófagos e hiperprodução de citocinas pró-inflamatórias, a chamada tempestade de citocinas. Nesse contexto, o estresse oxidativo e a inflamação que são cruciais para a defesa contra a infecção por COVID-19, tornam-se prejudiciais caso não sejam adequadamente regulados (D-Marco et al., 2020).

Em estudos sobre a SARS-CoV-2, a maioria das células que expressam as proteínas ECA2 e a SARS-CoV-2 foram reativas contra o Fator de Transformação do Crescimento Beta (TGF $\beta 1$ ), Fatores de Necrose Tumoral Alfa (TNF $\alpha$ ), interleucina (IL) $1 \beta$ e IL 6, sugerindo sua superprodução em pacientes infectados. Desta forma, a necrose tubular aguda foi demonstrada como a característica histopatológica mais comum da LRA em pacientes com SARS (Su et al., 2020).

Gupta et al. (2020) defende que o mecanismo que causa o apagamento do processo do pé podocitário é provavelmente mediado por várias vias imunológicas que envolvem desequilíbrio nas populações de células $\mathrm{T}$ durante a fase ativa da doença com uma prevalência de perfil de citocinas de células T auxiliares (Th2) tipo 2 (Th2; IL4,5,9,10 e 13). A tempestade de citocinas geradas por Th2 atuando como um gatilho para a podocitopatia, portanto, permanece uma possibilidade distinta (Gupta et al., 2020).

Além da síndrome da tempestade de citocinas induzida por sepse, pode ocorrer lesão celular direta nos rins por infecção por coronavírus (Cheng et al., 2020; Naicker et al., 2020; Xu et al., 2020). Su et al. (2020) mostraram que as células 
epiteliais viscerais tubulares e glomerulares são as células alvo primárias da SARS-CoV-2. Isso aumenta a propensão aos efeitos citopáticos do vírus em caso de viremia (Ulu et al., 2020).

Ademais, nesses pacientes pode ocorrer a presença de trombos de fibrina que apontem para o diagnóstico de microangiopatia trombótica. Esse estado hipercoagulável em pacientes com COVID-19 aumenta a probabilidade de trombos nos vasos sanguíneos pulmonares na microvasculatura do rim (Connors \& Levy, 2020). Também é possível que a oclusão trombótica de grandes vasos sanguíneos renais (por exemplo, artéria renal, veia renal) possa contribuir para lesão renal (Farouk et al., 2020; Rossi et al., 2020).

Outrossim, a rabdomiólise tem sido apontada como fator causal e/ou desencadeante de LRA na infecção pelo SARSCoV-2. A rabdomiólise é uma síndrome resultante da lesão do músculo esquelético e caracterizada pelo vazamento de proteínas e enzimas dos miócitos para a corrente sanguínea (Alrubaye \& Chouhary, 2020; Mukherjee, Ghosh \& Aftab, 2020; TAXBRO et al., 2020).

O mecanismo exato pelo qual os vírus causam destruição muscular não foi estabelecido, mas dois possíveis mecanismos foram propostos: necrose muscular relacionada à potencial invasão viral direta dos miócitos e danos imunomediados causados por citocinas miotóxicas (Mukherjee, Ghosh \& Aftab, 2020).

A mioglobinúria é patognomônica de rabdomiólise e as evidências sugerem que a mioglobina prejudica a filtração glomerular por meio de vários mecanismos, incluindo vasoconstrição intrarrenal, lesão isquêmica do túbulo e obstrução tubular (Bosch, Poch \& Grau, 2009). Diante disso, ocorre depleção de fluido intravascular por sequestro no músculo danificado e liberação de mediadores endógenos, resultando em redução do fluxo sanguíneo renal, como endotelina-1, tromboxanoA2, fator de necrose tumoral $\alpha$ e F2-isoprostanos. Esse quadro resulta coletivamente no desenvolvimento de LRA (Mukherjee, Ghosh \& Aftab, 2020). A IRA é uma complicação comum da rabdomiólise, acompanhando 7\%-10\% dos casos (Bosch, Poch \& Grau, 2009).

Em um estudo feito por Cui et al. (2020), os pacientes com LRA na COVID-19 apresentaram uma taxa de mortalidade significativamente mais alta. Os mesmos observaram que fenômenos semelhantes foram observados em pacientes com SARS em 2003, sendo a taxa de mortalidade significativamente maior entre pacientes com SARS e insuficiência renal aguda do que entre aqueles com SARS e sem insuficiência renal. Na análise de subgrupos, foi descoberto que o tempo de início da LRA caracterizou duas populações diferentes. Pacientes com LRA tardio (início 72 horas após a admissão) apresentaram uma taxa de mortalidade mais alta e tempo de internação significativamente maior do que pacientes com LRA precoce. Cui et al. (2020) postula que esse resultado pode ser devido ao fato de a LRA precoce ser provavelmente determinada, até certo ponto, por efeitos citopáticos diretos do vírus, mecanismos efetores imunológicos específicos induzidos por vírus, citocinas induzidas por vírus ou efeitos mediadores e hipoperfusão de órgãos hipovolêmicos, enquanto a LRA tardia geralmente aparecia em combinação com sepse, falência de múltiplos órgãos e uso de agentes nefrotóxicos. Em consonância com essa constatação, Tarragón et al. (2020) identificou que o fenótipo IRA intra-hospitalar apresenta proporção significativamente maior de necrose tubular aguda associada à sepse, enquanto o grupo IRA na admissão está associado principalmente a fatores pré-renais. Em geral, o grupo IRA intra-hospitalar concentra pacientes mais gravemente enfermos, com acometimento pulmonar mais grave, com necessidade de ventilação mecânica e internação em UTI.

Nota-se, portanto, que a etiologia clínica da IRA é muito variada. A primeira análise histológica de biópsias renais post-mortem de 26 pacientes da China mostrou lesão tubular aguda, colapso de glomerulopatia, lesão vascular e apagamento do processo podocitário do pé (Su et al., 2020). Em Tarragón et al. (2020) apenas 3 dos casos incluídos (7,3\%) estavam associados a doenças glomerulares. Os autores detectaram um caso até então desconhecido de nefropatia lúpica e 2 casos de microangiopatia trombótica, sendo esta última inerente à fisiopatologia renal descrita pela infecção por SARS-CoV-2 (Battle et al., 2020). 


\subsection{Perfil clínico dos pacientes com complicações renais agudas na COVID-19}

Quanto à história patológica prévia, estudos iniciais da China mostraram que hipertensão (63\%), diabetes (21\%) e doenças cardiovasculares (14\%) são comorbidades comuns em pacientes com COVID-19, especialmente naqueles com recuperação difícil (Li et al., 2020a).

Nos pacientes com hipertensão e doenças cardíacas, além da influência da própria doença na função renal, o tratamento contínuo também exerce efeito negativo. Isso porque medicamentos comumente usados para o tratamento dessas comorbidades como inibidores da enzima conversora de angiotensina (IECA) e bloqueadores do receptor de angiotensina (BRA), aumentam a expressão do gene ECA2, facilitando a invasão viral, e mitigam a regulação negativa da ECA2. Outros fármacos, como diuréticos e a administração de contraste iodado também contribuem na LRA (Chenna et al., 2020).

Clinicamente, os sintomas urinários podem estar presentes, inclusive, em pacientes sem sintomas agudos de COVID19 (Gupta et al., 2020). A diarreia é um fator relevante para a desidratação nesses pacientes. Embora tenha sido considerada um sintoma menor na infecção por SARS-CoV-2, presente em apenas 3-10\% dos casos (Huang et al., 2020; Wang et al., 2020a; Zhou et al., 2020), no estudo de Tarragón et al. (2020) com pacientes que apresentaram complicações renais a diarreia foi relatada em 48,8\% dos casos (Tarragón et al., 2020).

Pesquisas sugerem que o aumento de alguns parâmetros renais, como proteinúria, hematúria, ureia e creatinina sérica (SCr) se associam a mortalidade até cinco vezes maior neste grupo (Cheng et al., 2020; Li et al., 2020b; Moitinho et al., 2020). Pan et al. (2020) afirmam que os podócitos são particularmente vulneráveis a ataques virais e bacterianos, e o dano aos podócitos induz facilmente proteinúria intensa.

Entretanto, quando comparada a estudos anteriores a respeito da infecção por MERS-CoV, a infecção por SARSCoV-2 apresenta menos proteinúria (30,3\%), sugerindo que a lesão renal aguda e danos aos túbulos renais causados pelo SARSCoV-2 é menos severo quando comparado ao MERS-CoV (proteinúria em 60\% dos pacientes) (Cha et al., 2020; Na et al., 2020).

Nos casos em que há rabdomiólise, as características clínicas são inespecíficas e menos de $10 \%$ dos pacientes apresentam a tríade clássica de fraqueza, mialgia e urina cor de chá. Portanto, a suspeita clínica e o monitoramento rigoroso são os pilares para o diagnóstico de rabdomiólise (Bosch, Poch \& Grau, 2009; Zutt et al., 2014). Os achados laboratoriais incluem níveis elevados de creatina quinase, lactato desidrogenase e enzimas hepáticas e níveis aumentados de mioglobulina (Bosch, Poch \& Grau, 2009).

É importante destacar que pacientes com IRA intra-hospitalar em geral apresentam maior gravidade clínica e apresentaram maiores valores de ferritina, proteína C reativa, IL-6, LDH e dímero D do que aqueles que já estavam internados com IRA (Tarragón et al., 2020).

Quanto ao desfecho, a LRA está associada ao aumento da mortalidade em pacientes com COVID-19. A incidência de LRA foi de 23\% em 101 pacientes relatados por Shi et al. (2020), que morreram de infecção por COVID-19. Em Bajwa et al. (2020), a taxa de incidência de LRA em pacientes com COVID-19 foi de 6\%, menor que taxa de incidência de outras lesões de órgãos, como pulmões e coração, no COVID-19. No entanto, a taxa de mortalidade nesses pacientes alcançou alarmantes $94 \%$. Nesse sentido, a literatura aponta que BUN elevada, creatinina sérica basal elevada, pico de creatinina sérica $>1,5$, estágio $2 \mathrm{e}$ 3 de IRA, proteinúria e hematúria estão associados à mortalidade (Cheng et al., 2020).

Em Tarragón et al. (2020), 22\% dos pacientes morreram, 56\% tiveram alta sem necessidade de terapia renal substitutiva (TRS), 2,4\% com necessidade de TRS e 19,5\% continuaram internados. Esses resultados evidenciam a necessidade de que os pacientes propensos ao desenvolvimento de LRA recebam atenção especial e monitoramento frequente das funções renais. 


\subsection{Abordagem muldisciplinar das manifestações nefrológicas no contexto pandêmico}

No que diz respeito à prevenção da COVID-19, as medidas de contenção do contágio com coronavírus envolve não só médicos, enfermeiros, técnicos e demais profissionais da saúde, como a sociedade em geral. Nesse contexto, destaca-se também a importância dos governos e organizações da saúde construírem políticas provisórias que abordem o uso de equipamentos de proteção, reconhecimento de sinais e sintomas e como reagir após contato com indivíduo infectado, além de fornecer subsídios para o adequado manejo da doença (Ulu et al., 2020).

A gestão hospitalar também tem papel fundamental nesse processo. A onda inicial de COVID-19 já sobrecarregou os sistemas de saúde em todo o mundo, levantando discussões internacionais sobre ética de triagem, alocação de recursos escassos e tomada de decisões em cenário de crise (Mcguire et al., 2020).

Uma vez que a COVID-19 afeta múltiplos sistemas, especialistas das mais diversas áreas - cardiologia, nefrologia, gastroenterologia, entre outros - atuam sincronicamente no manejo do paciente infectado (Cheng et al., 2020; Hirsch et al., 2020). Os nefrologistas estão na segunda linha de frente, imediatamente após infectologistas, intensivistas e pneumologistas (Ulu et al., 2020).

Nos pacientes com LRA e COVID-19, o bom prognóstico do paciente implica em contenção dos outros fatores desencadeantes da doença, como depleção de volume, drogas nefrotóxicas e hipotensão, processo que demanda atenção multidisciplinar (LIPTON et al., 2020).

Nos pacientes com doença renal terminal (DRT), especialmente pacientes em hemodiálise (HD), o risco de complicações da COVID-19 é elevado. A própria uremia altera a imunidade inata e adaptativa, diante da disfunção de monócitos e neutrófilos, maturação diminuída de células $\mathrm{T}$ e menor resposta humoral, configurando quadro de imunossupressão. Os serviços de HD podem ser sobrecarregados pela demanda aguda, não conseguindo corresponder às necessidades de recursos de diálise e pessoal (Parsons \& Martin, 2020). Essa demanda soma-se a dos pacientes crônicos, haja vista que as indicações para início e manutenção da diálise permanecem as mesmas para pacientes infectados ou não pelo coronavírus (Betonico, Lima \& Tome, 2020).

Lipton et al. (2020) identificou em Nova Iorque que a ampla demanda na nefrologia, diante das implicações da COVID-19 nesse sistema, nefrologistas pediátricos fossem alocados para nefrologia de adultos. Além disso, destacou também que diversos pediatras transitaram para o atendimento em UTI. Goldfarb et al. (2020) reitera que esforços conjuntos equilibrados nos centros médicos devem ocorrer de forma a manter a capacidade de fornecer TRS aos pacientes agudos e crônicos.

De acordo com a nota técnica e orientações clínicas sobre a Injúria Renal Aguda em pacientes com COVID-19 da Sociedade Brasileira de Nefrologia e Associação de Medicina Intensiva Brasileira, na atual conjuntura é de fundamental importância a estreita colaboração entre intensivistas e nefrologistas na observação à beira do leito de parâmetros hemodinâmicos e avaliação do estado volêmico (Suassuna et al., 2020).

Nesse contexto, o nefrologista pode ser acionado mesmo em graus discretos de disfunção renal. Apesar da recomendação inicial de limitar a entrada de nefrologistas e da enfermagem de diálise no atendimento de pacientes com COVID-19 a fim de evitar exposição e infecção desses profissionais, em muitos centros o excesso de pacientes infectados complicados por IRA sobrecarregou as equipes de cuidados intensivos, o que alterou os protocolos. Recomenda-se agora a participação direta do nefrologista e da enfermagem de nefrologia no atendimento a esses pacientes (Suassuna et al., 2020).

\section{Conclusão}

O SARS-CoV-2 invade o sistema geniturinário com relativa facilidade, em especial em pacientes com comorbidades, e causa dano direto ou por meio da desregulação de mecanismos imunológicos, o que ocasiona um quadro clínico que mescla 
sintomas de falência renal e quadro infeccioso. Considerando a frequente evolução da COVID-19 para LRA e os impactos dessa associação nos sistemas de saúde, é imprescindível a coesão entre equipe de multiprofissional e gestão em saúde.

Visto tratar-se de uma temática explorada apenas recentemente, mas com grande repercussão nos sistemas de saúde, sugere-se a realização de ensaios clínicos controlados que avaliem a relação entre COVID-19 e manifestações renais, a fim de fornecer dados com mais elevados níveis de evidência.

\section{Referências}

Alrubaye, R., \& Choudhary, H. (2020). Severe Rhabdomyolysis in a 35-Year-old Woman with COVID-19 due to SARS-CoV-2 Infection: A Case Report. The American Journal of Case Reports, 21, e926733-1.

Bajwa, H., Riaz, Y., Ammar, M., Farooq, S., \& Yousaf, A. (2020). The dilemma of renal involvement in COVID-19: a systematic review. Cureus, 12(6), e8632.

Betonico, G. N., Lima, E. Q., \& Tome, A. C. N. (2020). Challenges in COVID-19 medical response: a nephrology perspective. Eur J Clin Invest, e13328.

Bosch, X., Poch, E., \& Grau, J. M. (2009). Rhabdomyolysis and acute kidney injury. New England Journal of Medicine, 361(1), 62-72.

Cha, R. H., Joh, J. S., Jeong, I., Lee, J. Y., Shin, H. S., Kim, G., \& Kim, Y. (2015). Renal complications and their prognosis in Korean patients with Middle East respiratory syndrome-coronavirus from the central MERS-CoV designated hospital. Journal of Korean medical science, $30(12), 1807-1814$.

Cheng, Y., Luo, R., Wang, K., Zhang, M., Wang, Z., Dong, L., Li, J., Yao, Y., Ge, S., \& Xu, G. (2020). Kidney disease is associated with in-hospital death of patients with COVID-19. Kidney international, 97(5), 829-838.

Chenna, A., Konala, V. M., Bose, S., Roy, S., Madhira, B. R., Gayam, V., Naramala, S., \& Adapa, S. (2020). Acute Kidney Injury in a Case Series of Patients with Confirmed COVID-19 (Coronavirus Disease 2019): Role of Angiotensin-Converting Enzyme 2 and Renin-Angiotensin System Blockade. Case reports in nephrology, 2020.

Connors, J. M., \& Levy, J. H. (2020). Thromboinflammation and the hypercoagulability of COVID-19. Journal of Thrombosis and Haemostasis, 18(7), 15591561 .

Cui, X., Yu, X., Wu, X., Huang, L., Tian, Y., Huang, X., Zhang, Z., Cheng, Z., Guo, Q., Zhang, Y., Cai, Y., \& Zhan, Q. (2020). Acute kidney injury in patients with the coronavirus disease 2019: a multicenter study. Kidney and Blood Pressure Research, 45(4), 612-622.

D’Marco, L., Puchades, M. J., Romero-Parra, M., Gimenez-Civera, E., Soler, M. J., Ortiz, A., \& Gorriz, J. L. (2020). Coronavirus disease 2019 in chronic kidney disease. Clinical kidney journal, 13(3), 297-306.

Farouk, S. S., Fiaccadori, E., Cravedi, P., \& Campbell, K. N. (2020). COVID-19 and the kidney: what we think we know so far and what we don't. Journal of nephrology, 1-6.

Goldfarb, D. S., Benstein, J. A., Zhdanova, O., Hammer, E., Block, C. A., Caplin, N. J., Thompson, N., \& Charytan, D. M. (2020). Impending shortages of kidney replacement therapy for COVID-19 patients. Clinical Journal of the American Society of Nephrology, 15(6), 880-882.

Gupta, R. K., Bhargava, R., Shaukat, A. A., Albert, E., \& Leggat, J. (2020). Spectrum of podocytopathies in new-onset nephrotic syndrome following COVID19 disease: a report of 2 cases. BMC nephrology, 21(1), 1-7.

Hirsch, J. S., Ng, J. H., Ross, D. W., Sharma, P., Shah, H. H., Barnett, R. L., Hazzan, A. D., Fishbane, S., Jhaveri, K. D., \& Northwell COVID-19 Research Consortium. (2020). Acute kidney injury in patients hospitalized with COVID-19. Kidney international, 98(1), 209-218.

Hoffmann, M., Kleine-Weber, H., Schroeder, S., Krüger, N., Herrler, T., Erichsen, S., Erichsen, S., Schiergens, T. S., Herrler, G., Wu, N. W., Nitsche, A., Müller, M. A., Drosten, C., \& Pöhlmann, S. (2020). SARS-CoV-2 cell entry depends on ACE2 and TMPRSS2 and is blocked by a clinically proven protease inhibitor. cell, 181(2), 271-280.

Li, B., Yang, J., Zhao, F., Zhi, L., Wang, X., Liu, L., Bi, Z., \& Zhao, Y. (2020a). Prevalence and impact of cardiovascular metabolic diseases on COVID-19 in China. Clinical Research in Cardiology, 109(5), 531-538.

Li, Z., Wu, M., Yao, J., Guo, J., Liao, X., Song, S., Li, J., Duan, G., Zhou, Y., Wu, X., Zhou, Z., Wang, T., Hu, M., Chen, X., Fu, Y., Lei, C., Dong, H., Xu, C., Hu, Y., Han, M., Zhou, Y., Jia, H., Chen, X., \& Yan, J. (2020b). Caution on kidney dysfunctions of COVID-19 patients. MedRxiv. http://dx.doi.org/10.2139/ssrn.3559601.

Lipton, M., Kavanagh, C. R., Mahajan, R., Jain, N. G., Uy, N. S., Dogra, S., \& Lin, F. (2020). Role of pediatric nephrologists in managing adults with AKI due to COVID-19. Pediatric Nephrology, 35(11), 2019-2022.

McGuire, A. L., Aulisio, M. P., Davis, F. D., Erwin, C., Harter, T. D., Jagsi, R., Klitzman, R., Macauley, R., Racine, E., Wolf, S., M., Wynia, M., Wolpe, P. R., \& COVID-19 Task Force of the Association of Bioethics Program Directors (ABPD). (2020). Ethical challenges arising in the COVID-19 pandemic: An overview from the Association of Bioethics Program Directors (ABPD) task force. The American Journal of Bioethics, 20(7), 15-27.

Moitinho, M. S., Belasco, A. G. D. S., Barbosa, D. A., \& Fonseca, C. D. D. (2020). Acute Kidney Injury by SARS-CoV-2 virus in patients with COVID-19: an integrative review. Revista brasileira de enfermagem, 73.

Mukherjee, A., Ghosh, R., \& Aftab, G. (2020). Rhabdomyolysis in a patient with coronavirus disease 2019. Cureus, 12(7). 
Na, K. R., Kim, H. R., Ham, Y., Choi, D. E., Lee, K. W., Moon, J. Y., Kim, Y. S., Cheon, S., Sohn, K. M., Kim, J., Kim, S., Jeong, H., \& Jeon, J. W. (2020). Acute kidney injury and kidney damage in COVID-19 patients. Journal of Korean medical science, 35(28), e257.

Pan, X. W., Xu, D., Zhang, H., Zhou, W., Wang, L. H., \& Cui, X. G. (2020). Identification of a potential mechanism of acute kidney injury during the COVID-19 outbreak: a study based on single-cell transcriptome analysis. Intensive care medicine, 46(6), 1114-1116.

Pan, X. W., Xu, D., Zhang, H., Zhou, W., Wang, L. H., \& Cui, X. G. (2020). Identification of a potential mechanism of acute kidney injury during the COVID-19 outbreak: a study based on single-cell transcriptome analysis. Intensive care medicine, 46(6), 1114-1116.

Parsons, J. A., \& Martin, D. E. (2020). A call for dialysis-specific resource allocation guidelines during COVID-19. The American Journal of Bioethics, 20(7), 199-201.

Pereira A. S., Shitsuka D. M., Parreira, F. J., \& Shitsuka, R. (2018). Metodologia da pesquisa científica. UFSM. https://www.ufsm.br/app/uploads/sites/358/2019/02/ Metodologia-da-Pesquisa-Cientifica_final.pdf em: 14/03/2021.

Richardson, S., Hirsch, J. S., Narasimhan, M., Crawford, J. M., McGinn, T., Davidson, K. W., \& Northwell COVID-19 Research Consortium. (2020). Presenting characteristics, comorbidities, and outcomes among 5700 patients hospitalized with COVID-19 in the New York City area. Jama, 323(20), 20522059

Rossi, G. M., Delsante, M., Pilato, F. P., Gnetti, L., Gabrielli, L., Rossini, G., Parenti, E., Maccari, C., Greco, P., Mario, F. D., Maggiore, U., Regolisti, G., \& Fiaccadori, E. (2020). Kidney biopsy findings in a critically ill COVID-19 patient with dialysis-dependent acute kidney injury: a case against "SARS-CoV-2 nephropathy". Kidney international reports, 5(7), 1100-1105.

Shi, Q., Zhao, K., Yu, J., Jiang, F., Feng, J., Zhao, K., Zhang, X., Chen, X., Hu, P., Hong, Y., Li, M., Liu, F., Chen, C., \& Wang, W. (2020). Clinical characteristics of 101 COVID-19 nonsurvivors in Wuhan, China: a retrospective study. MedRxiv. https://doi.org/10.1101/2020.03.04.20031039.

Simões e Silva, A. C., Silveira, K. D., Ferreira, A. J., \& Teixeira, M. M. (2013). ACE2, angiotensin-(1-7) and M as receptor axis in inflammation and fibrosis. British journal of pharmacology, 169(3), 477-492.

Su, H., Yang, M., Wan, C., Yi, L. X., Tang, F., Zhu, H. Y., Yi, F., Yang, H., Fogo, A. B., Nie, X., \& Zhang, C. (2020). Renal histopathological analysis of 26 postmortem findings of patients with COVID-19 in China. Kidney international, 98(1), 219-227.

Suassuna, J. H. R., Lima, E. Q. D., Rocha, E., Castro, A., Burdmann, E. D. A., Carmo, L. P. D. F. D., Yu, L., Ibrahim, M. Y., Betônico, G. N., Neto, A. L. C., Ávila, M. O. N., Gonçalves, A. R. R., Costa, C. B. S., Bresolin, N. L., Abreu, A. P., Lobo, S. M. A., \& Nascimento, M. M. D. (2020). Nota técnica e orientações clínicas sobre a Injúria Renal Aguda (IRA) em pacientes com Covid-19: Sociedade Brasileira de Nefrologia e Associação de Medicina Intensiva Brasileira. Brazilian Journal of Nephrology, 42(2), 22-31.

Taxbro, K., Kahlow, H., Wulcan, H., \& Fornarve, A. (2020). Rhabdomyolysis and acute kidney injury in severe COVID-19 infection. BMJ Case Reports CP, 13(9), e237616.

Ulu, S., Gungor, O., Gok Oguz, E., Hasbal, N. B., Turgut, D., \& Arici, M. (2020). COVID-19: a novel menace for the practice of nephrology and how to manage it with minor devastation?. Renal failure, 42(1), 710-725.

Wang, D., Hu, B., Hu, C., Zhu, F., Liu, X., Zhang, J., Wang, B., Xiang, H., Cheng, Z., Xiong, Y., Zhao, Y., Li, Y., Wang, X., \& Peng, Z. (2020a). Clinical characteristics of 138 hospitalized patients with 2019 novel coronavirus-infected pneumonia in Wuhan, China. Jama, 323(11), 1061-1069.

Wang, L., Li, X., Chen, H., Yan, S., Li, D., Li, Y., \& Gong, Z. (2020b). Coronavirus disease 19 infection does not result in acute kidney injury: an analysis of 116 hospitalized patients from Wuhan, China. American journal of nephrology, 51(5), 343-348.

Wrapp, D., Wang, N., Corbett, K. S., Goldsmith, J. A., Hsieh, C. L., Abiona, O., Graham, B. S., \& McLellan, J. S. (2020). Cryo-EM structure of the 2019nCoV spike in the prefusion conformation. Science, 367(6483), 1260-1263.

Yang, X., Yu, Y., Xu, J., Shu, H., Xia, J., Liu, H., Wu, Y., Zhang, L., Yu, Z., Fang, M., Yu, T, Wang, Y., Pan, S., Zou, X., Yuan, S., \& Shang, Y. (2020). Clinical course and outcomes of critically ill patients with SARS-CoV-2 pneumonia in Wuhan, China: a single-centered, retrospective, observational study. The Lancet Respiratory Medicine, 8(5), 475-481.

Zhang, G., Hu, C., Luo, L., Fang, F., Chen, Y., Li, J., Peng, Z., \& Pan, H. (2020). Clinical features and short-term outcomes of 221 patients with COVID-19 in Wuhan, China. Journal of Clinical Virology, 127, 104364.

Zhou, F., Yu, T., Du, R., Fan, G., Liu, Y., Liu, Z., Xiang, J., Wang, Y., Song, B., Gu, X., Guan, L., Wei, Y., Li, H., Wu, X., Xu, J., Tu, S., Zhang, Y., \& Cao, B. (2020). Clinical course and risk factors for mortality of adult inpatients with COVID-19 in Wuhan, China: a retrospective cohort study. The lancet, 395(10229), 1054-1062.

Zou, X., Chen, K., Zou, J., Han, P., Hao, J., \& Han, Z. (2020). Single-cell RNA-seq data analysis on the receptor ACE2 expression reveals the potential risk of different human organs vulnerable to 2019-nCoV infection. Frontiers of medicine, 14, 185-192.

Zutt, R., Van Der Kooi, A. J., Linthorst, G. E., Wanders, R. J. A., \& De Visser, M. (2014). Rhabdomyolysis: review of the literature. Neuromuscular Disorders, 24(8), 651-659. 